\title{
Application of Centrifugal Partition Chromatography for Bioactivity-Guided Purification of Antioxidant-Response-Element-Inducing Constituents from Atractylodis Rhizoma Alba
}

\author{
Myeong Il Kim ${ }^{1,+}$, Ji Hoon Kim ${ }^{1,+}$, Ahmed Shah Syed ${ }^{1,2}$, Young-Mi Kim ${ }^{1}$, \\ Kevin Kyungsik Choe ${ }^{1}$ and Chul Young Kim ${ }^{1, *(D)}$ \\ 1 College of Pharmacy and Institute of Pharmaceutical Science and Technology, Hanyang University, \\ Ansan, Gyeonggi-do 15588, Korea; auddlf1117@hanmail.net (M.I.K.); gg890718@gmail.com (J.H.K.); \\ shahahmed454@gmial.com (A.S.S); ymikim12@hanyang.ac.kr (Y.-M.K.); kkchoe@hanyang.ac.kr (K.K.C.) \\ 2 Department of Pharmacognosy, Faculty of Pharmacy, University of Sindh, Jamshoro 76080, Pakistan \\ * Correspondence: chulykim@hanyang.ac.kr; Tel.: +82-31-400-5809; Fax: +82-31-400-5958 \\ + These authors contributed equally to this work.
}

Received: 3 August 2018; Accepted: 5 September 2018; Published: 6 September 2018

\begin{abstract}
Activity-guided separation of antioxidant response element (ARE)-inducing constituents from the rhizomes of Atractylodis Rhizoma Alba was performed by the combination of centrifugal partition chromatography (CPC) and an ARE luciferase reporter assay. From $3 \mathrm{~g}$ of the active $n$-hexane fraction, one polyacetylene, $(6 E, 12 E)$-tetradeca-6,12-dien-8,10-diyne-1,3-diyl diacetate $(47.3 \mathrm{mg})$, and two sesquiterpenes, atractylenolide I (40.9 mg), and selina-4(14),7(11)-dien-8-one $(6.0 \mathrm{mg})$ were successfully isolated by CPC with $n$-hexane-ethyl acetate-methanol-water $(8: 2: 8: 2, \mathrm{v} / \mathrm{v})$. The chemical structures of the isolated compounds were determined by ${ }^{1} \mathrm{H}-$ and ${ }^{13} \mathrm{C}-\mathrm{NMR}$ and ESI-MS. Among the isolated compounds, (6E,12E)-tetradeca-6,12-diene-8,10-diyne-1,3-diol diacetate and selina-4(14),7(11)-dien-8-one increased ARE activity 32.9-fold and 16.6-fold, respectively, without significant cytotoxicity, when $5 \mu \mathrm{M}$ sulforaphane enhanced ARE activity 27.1-fold. However, atractylenolide I did not increase ARE activity at $100 \mu \mathrm{M}$, and showed cytotoxicity at concentrations over $10 \mu \mathrm{M}$.
\end{abstract}

Keywords: Atractylodis Rhizoma Alba; centrifugal partition chromatography; antioxidant response element; polyacetylene; sesquiterpenes

\section{Introduction}

Oxidative stress is involved in various pathological conditions including ageing, cancer, inflammation, and chronic diseases [1]. Reactive oxygen species can be scavenged by the use of antioxidants including vitamins, carotenoids, and polyphenolic compounds [2]. Induction of antioxidant signaling pathway via upregulation of endogenous antioxidant/phase II detoxifying enzymes may be an alternative defensive approach. The activation of nuclear factor erythroid 2-related factor 2 (Nrf2)-antioxidant response element (ARE) pathway leads to the induction of antioxidant/phase II detoxifying enzymes including heme oxygenase 1, NAD $(\mathrm{P}) \mathrm{H}$ : quinine oxidoreductase 1 , and glutathione $S$-transferase. There is growing interest in molecules that activate the Nrf2-ARE signaling pathway [3].

Atractylodis Rhizoma Alba (the rhizome of Atractylodes macrocephala Koidz. or A. japonica Koidz. ex Kitam.) is a traditional medicine in Korea, Japan, and China. In Korea, the aerial parts of Atractylodis Rhizoma Alba are eaten as vegetables, while the rhizomes are used as medicinal herbs. According to 
Korean traditional medicine publications, Atractylodis Rhizoma Alba has warm properties and a bitter taste; it strengthens the stomach and spleen, resolves dampness, and stops sweating [4,5].

Previous phytochemical investigations led to the isolation of sesquiterpenes, polyacetylenes and phenolic acids from Atractylodis Rhizoma Alba, which have various biological effects such as antioxidants, gastroprotective and anti-inflammatory activities, as well as inhibiting aromatase and the farnesoid $\mathrm{X}$ receptor and modulating progesterone receptors [6-12].

The separation and purification of the bioactive molecules from natural products requires repeated chromatography, and in some cases the active ingredient does not elute by absorption to the solid phase [13]. To overcome these problems, centrifugal partition chromatography (CPC) is a viable alternative method. CPC is a preparative, support-free, liquid-liquid chromatographic technique, which is an efficient tool for bioactivity-guided isolation of natural ingredients that allows increased sample loading and the absence of sample loss due to irreversible sample absorption to a solid column [14-18].

The aim of this study was to develop a simple activity-guided isolation procedure for ARE-inducing compounds from the crude extract of A. macrocephala by CPC and an ARE luciferase reporter assay in HepG2 cells. As a result, two sesquiterpenes including atractylenolide I (1) and selina4(14),7(11)-dien-8-one (3), and one polyacetylene, (6E,12E)-tetradeca-6,12-diene-8,10-diyne-1,3-diol diacetate (2) (Figure 1) were obtained by one-step CPC. The ARE induction activity and cytotoxicity of these compounds 1-3 were also evaluated.<smiles>C=C1CCC[C@]2(C)C=C3OC(=O)C(C)=C3CC12</smiles>

Atractylenolide I (1)<smiles>C=C1CCC[C@]2(C)CC(=O)C(=C(C)C)CC12</smiles>

Selina-4(14),7(11)-dien-8-one (3)<smiles>CC=CC#CC#CC=CCCC(CCOC(C)=O)OC(C)=O</smiles>

(6E,12E)-Tetradeca-6,12-diene-8,10-diyne-1,3-diol diacetate (2)

Figure 1. Chemical structures of purified compounds 1-3.

\section{Results and Discussion}

\subsection{ARE Luciferase Assay and HPLC Analysis of the Crude Extract and Sub-Fractions}

Based on the ARE luciferase assay, the $n$-hexane fraction was the most active, followed by the ethyl acetate fraction. At a concentration of $30 \mu \mathrm{g} / \mathrm{mL}$, the $n$-hexane and ethyl acetate fractions enhanced ARE activity 27.1 and 17.1-fold, respectively. Sulforaphane, the positive control, increased ARE activity 49.9-fold at a concentration of $5 \mu \mathrm{M}$ (Figure 2A).

HPLC analysis of crude samples revealed that compounds 1-3 were major constituents of A. macrocephala in the $n$-hexane fraction (Figure 2B). Based on its ARE-enhancing activity and HPLC chromatogram, the $n$-hexane fraction was selected to purify active molecules by CPC. 
A

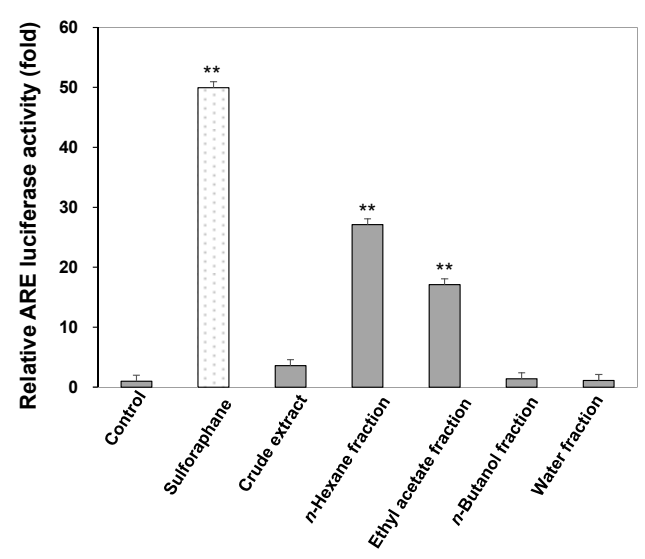

B

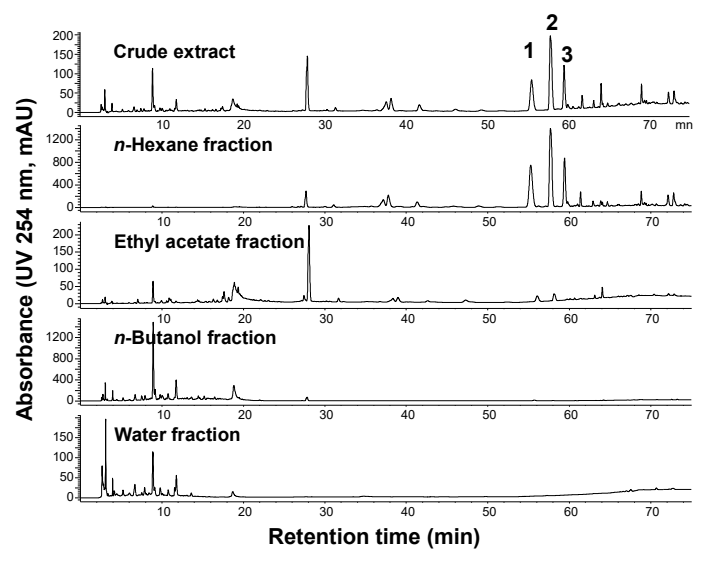

Figure 2. ARE luciferase activities in HepG2 cells (A) and HPLC chromatograms of the crude extract and its sub-fractions (B). The relative ARE luciferase activities were measured in the lysates of HepG2 cells that were stably transfected with pGL4.37 after exposure to $30 \mu \mathrm{g} / \mathrm{mL}$ of crude extract or sub-fractions for $12 \mathrm{~h}$. Data are presented as means \pm S.E. $(n=5)$. ${ }^{* *} p<0.01$ (compared with the vehicle-treated control).

\subsection{Optimization of the Two-Phase Solvent System and CPC Operation}

A two-phase solvent system with petroleum $\left(60-90{ }^{\circ} \mathrm{C}\right)$-ethyl acetate-ethanol-water $(4: 1: 4: 1, v / v)$ has previously been reported for the separation of actractylon and atractylenolide III from A. macrocephala by high-speed counter-current chromatography [19]. Based on this result, several two-phase solvent systems consisting of $n$-hexane-ethyl acetate-methanol-water at different ratios were tested to calculate $K$-values for major constituents of the $n$-hexane fraction (Table 1 ).

Table 1. Partition coefficients $(K)$ of $n$-hexane fraction of $A$. macrocephala.

\begin{tabular}{cccc}
\hline \multirow{2}{*}{$\begin{array}{c}\text { Solvent Systems } \\
\text { (HEMW, } \boldsymbol{v} / \boldsymbol{v})\end{array}$} & Atractylenolide I (1) & $\begin{array}{c}\text { K-VE,12E)-Tetradeca-6,12- } \\
\text { dien-8,10-diyne-1,3-diyl diacetate (2) }\end{array}$ & Selina-4(14),7(11)-dien-8-one (3) \\
\hline $9: 1: 9: 1$ & 0.82 & 0.40 & 1.93 \\
$8: 2: 8: 2$ & 1.54 & 0.86 & 3.62 \\
$7: 3: 7: 3$ & 3.37 & 2.35 & 7.80 \\
\hline
\end{tabular}

The $K$-value is defined as the peak area of the compounds in upper stationary phase divided by the peak area of those in the lower mobile phase. H: $n$-hexane, E: ethyl acetate, M: methanol, W: water.

CPC was performed with a solvent ratio of 8:2:8:2 $(v / v)$, although the $K$-value was better at a ratio of 9:1:9:1 $(v / v)$ for the three major compounds 1-3 in the $n$-hexane fraction. The peak at $28.6 \mathrm{~min}$, a major component in ethyl acetate fraction, was also expected to exert ARE-inducing activity (Figure 1). Therefore, this peak is more polar than compounds $\mathbf{1}-\mathbf{3}$, which is why the two-phase solvent system with 8:2:8:2 was more suitable for polar conditions than the 9:1:9:1 system.

The retention time of each compound 1-3 in CPC was calculated using a general chromatographic retention equation $\left(V_{R}=V_{M}+K_{C} V_{S}, V_{R}\right.$ : the retention volume of the solute, $V_{M}$ : the mobile phase volume, $K_{C}$ : the partition coefficient, $V_{S}$ : the stationary volume) [20]. In this experiment, SCPC-1000 was used (column volume: $1000 \mathrm{~mL}$, flow rate: $10 \mathrm{~mL} / \mathrm{min}$, mobile phase retention: 54\%). Therefore, the calculated retention time for each peak was $102.16 \mathrm{~min}$ (peak A), $140.24 \mathrm{~min}$ (peak B), and 251.12 min (peak C).

As shown in Figure 3, the $n$-hexane fraction $(3 \mathrm{~g})$ separated excellently into three major response CPC peak fractions: A (90-110 min), B (120-146 min), and C (178-227 min) (Table 2). The first two peaks (A and B) eluted as expected, but the third peak (C) eluted earlier than the calculated time. 
This difference is due to the gradual outflow of the stationary phase, which can be observed by a reduction in the operating pressure (Figure $3 \mathrm{~A}$ ).

A
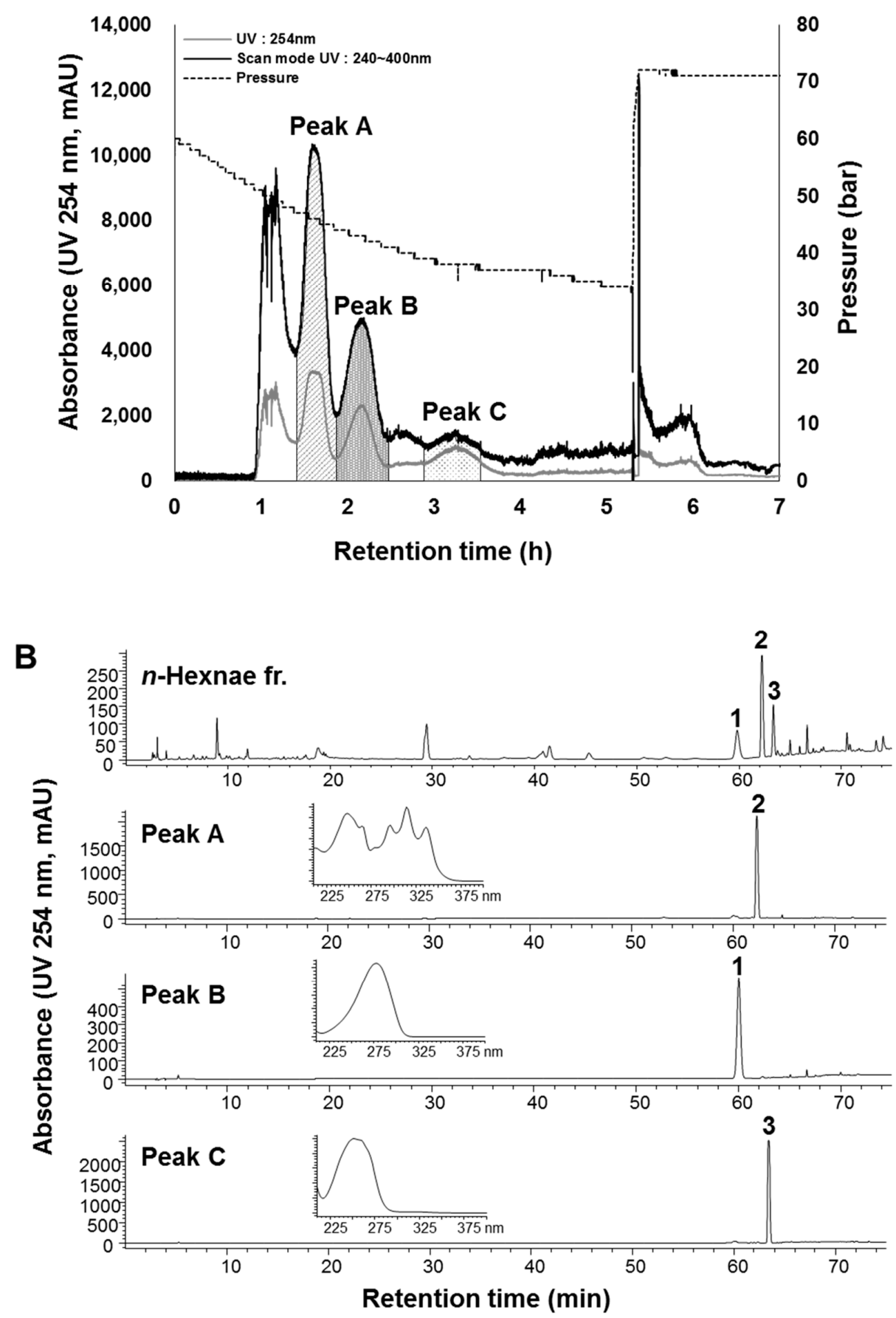

Figure 3. CPC chromatogram of the $n$-hexane fraction (A) and HPLC chromatograms and UV spectra of the $n$-hexane fraction and purified peaks (A-C) (B). CPC conditions: two-phase solvent system, $n$-hexane-ethyl acetate-methanol-water (8:2:8:2, $v / v)$, descending mode; mobile phase, lower (more hydrophilic) phase; flow rate, $10 \mathrm{~mL} / \mathrm{min}$; rotation speed, $1100 \mathrm{rpm}$; monitored at $254 \mathrm{~nm}$ and scan mode (240-400 nm). Peak A: (6E,12E)-tetradeca-6,12-diene-8,10-diyne-1,3-diol diacetate (2), peak B: atractylenolide I (1) and peak C: selina-4(14),7(11)-dien-8-one (3). 
Each CPC peak fraction from A to $\mathbf{C}$ was concentrated to yield $47.3 \mathrm{mg}$ of peak $\mathbf{A}, 40.9 \mathrm{mg}$ of peak $\mathbf{B}$ and $6.0 \mathrm{mg}$ of peak C. Afterward, the fractions obtained from the CPC chromatogram were analyzed via analytical HPLC. Fraction peaks A-C from the CPC with a descending mode corresponded to the peak order of 2, 1 and 3 in the HPLC chromatogram in Figure 3. The purities of atractylenolide I (1), (6E,12E)-tetradeca-6,12-dien-8,10-diyne-1,3-diyl diacetate (2) and selina-4(14),7(11)-dien-8-one (3) were demonstrated to be $>99 \%, 95 \%$ and $97 \%$, respectively, according to the area of the HPLC peaks at $254 \mathrm{~nm}$ (Figure 3B).

Table 2. Comparison of CPC retention time calculated by $K$-values and operation result in the two-phase solvent system of $n$-hexane-ethyl acetate-methanol-water $(8: 2: 8: 2, v / v)$.

\begin{tabular}{|c|c|c|c|}
\hline CPC Peaks & Peak A & Peak B & Peak C \\
\hline Compounds 1-3 & $\begin{array}{c}\text { (6E,12E)-tetradeca-6,12-dien- } \\
\text { 8,10-diyne-1,3-diyl diacetate (2) }\end{array}$ & atractylenolide I (1) & selina-4(14),7(11)-dien-8-one (3) \\
\hline$K$-values of compounds $1-3$ & 0.86 & 1.54 & 3.62 \\
\hline Retention time (calculated) & $102.16 \mathrm{~min}$ & $140.24 \mathrm{~min}$ & $251.12 \mathrm{~min}$ \\
\hline $\begin{array}{l}\text { Retention time (experimental } \\
\text { data) }\end{array}$ & $90-10 \mathrm{~min}$ & $120-146 \mathrm{~min}$ & $178-227 \mathrm{~min}$ \\
\hline
\end{tabular}

CPC operating conditions: CPC volume, $1000 \mathrm{~mL}$; flow rate, $10 \mathrm{~mL} / \mathrm{min}$; mobile phase retention, $54 \%$.

\subsection{ARE-Activating Effect of Purified Compounds 1-3}

The capability of the isolated compounds 1-3 to augment ARE activity was assessed by a luciferase assay in HepG2 cells. (6E,12E)-Tetradeca-6,12-diene-8,10-diyne-1,3-diol diacetate (2) and selina-4(14),7(11)-dien-8-one (3) enhanced ARE activity in a dose-dependent manner (Figure 4A) without significant cytotoxicity. At $100 \mu \mathrm{M}$, compounds 2 and 3 increase ARE activity 32.9-fold and 16.6-fold, respectively, without significant cytotoxicity, when $5 \mu \mathrm{M}$ sulforaphane enhanced ARE activity 27.1-fold. However, atractylenolide I (1) did not increase ARE activity at $100 \mu \mathrm{M}$, and showed cytotoxicity at concentrations over $10 \mu \mathrm{M}$ (Figure $4 \mathrm{~B}$ ).

In previous studies, $(6 E, 12 E)$-tetradeca-6,12-diene-8,10-diyne-1,3-diol diacetate (2) exhibited matrix metalloproteinase-13 downregulating activity in IL-1 $\beta$-treated SW1353 chondrocytes [21] and anti-methicillin-resistant Staphylococcus aureus activity [22], and selina-4(14),7(11)-dien-8-one (3) inhibited melanogenesis via reduction of expression of the melanogenic protein that regulated the mRNA level of tyrosinase and tyrosinase-related proteins 1 and -2 [6].

Our results suggest that $(6 E, 12 E)$-tetradeca-6,12-diene-8,10-diyne-1,3-diol diacetate (2) and selina-4(14),7(11)-dien-8-one (3) might reduce oxidative stress by enhancing cellular antioxidant systems without cytotoxicity and compounds $\mathbf{2}$ and $\mathbf{3}$ were major active molecules for ARE induction activity in $n$-hexane extract of $A$. macrocephala. 
A

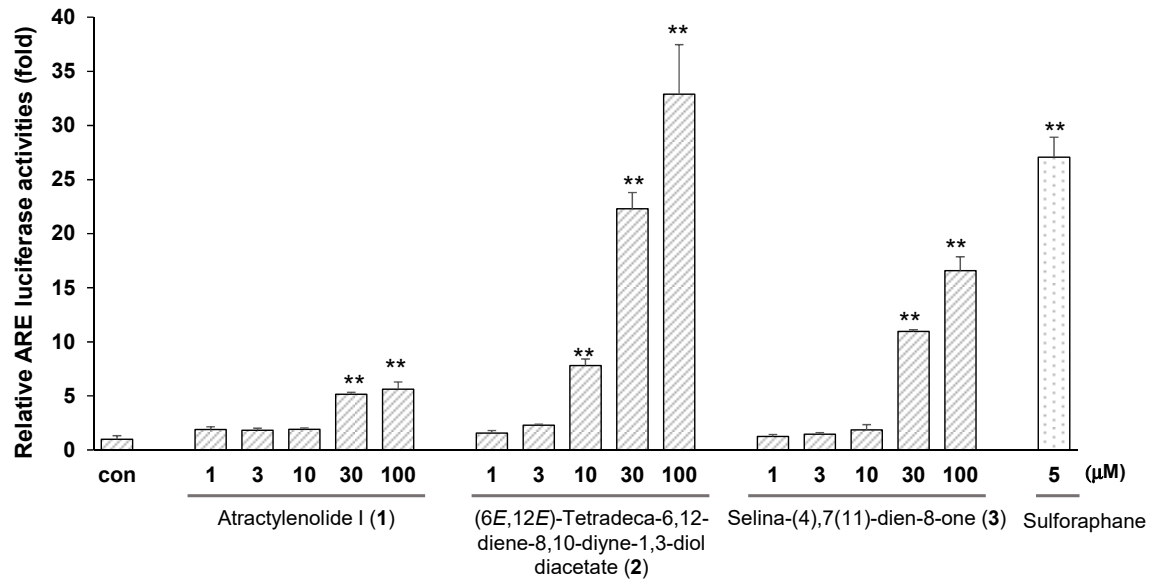

B

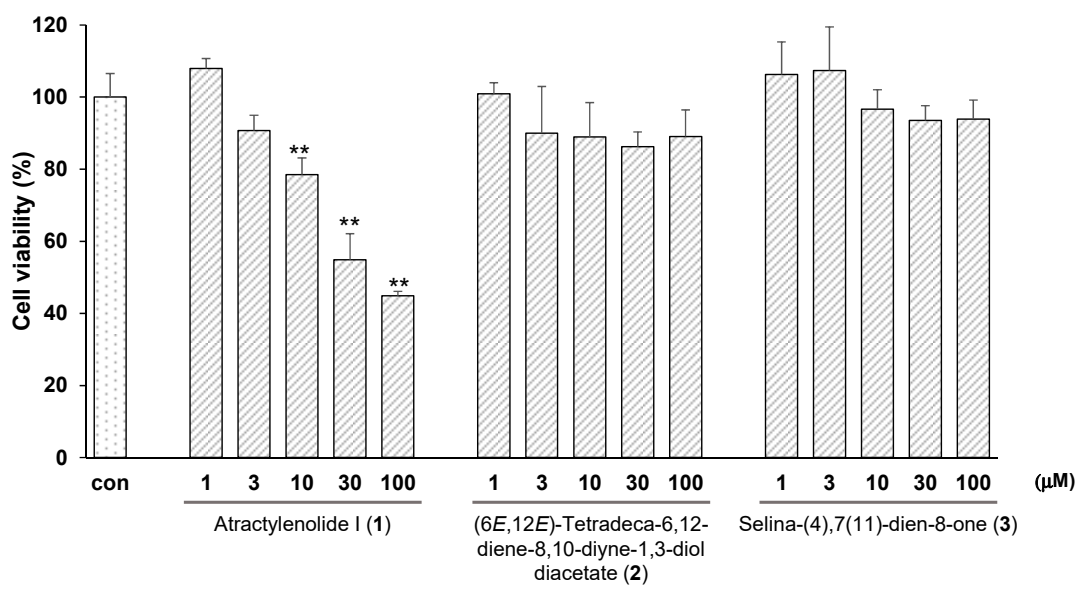

Figure 4. ARE luciferase activities (A) and cytotoxicity (B) of isolated compounds 1-3 in HepG2 cells. The relative ARE luciferase activities were measured in the lysates of HepG2 cells that were stably transfected with pGL4.37 after exposure to 1-100 $\mu \mathrm{M}$ of each compound for $12 \mathrm{~h}$. Cell viability was measured by the MTT assay. Data are presented as means \pm S.E. $(n=5) .{ }^{* *}, p<0.01$ (compared with the vehicle-treated control).

\section{Materials and Methods}

\subsection{Apparatus and Materials}

CPC was performed on an Armen fully integrated SCPC-100+1000 CPC spot instrument (Armen Instruments, St-Ave, France). This instrument is a fully automated system consisting of a CPC column compartment $(1000 \mathrm{~mL}$ rotor made of 21 stacked disks with a total of 1512 twin cells), a pump, an injector, a UV/vis detector, a fraction collector, a digital screen flat PC, and Armen Glider CPC software. The HPLC analysis was performed by an Agilent 1260 HPLC system (Agilent Technologies, Palo Alto, CA, USA): G1312C binary pump, a G1329B autosampler, a G1315D DAD detector, a G1316A column oven and ChemStation software. HPLC-grade solvents were purchased from Fisher Scientific (Pittsburgh, PA, USA). All other organic solvents used for extraction and CPC operation were analytical grade and obtained from Daejung (Gyonggi-do, Korea).

\subsection{Plant Material and Preparation of Crude Extracts}

The dried rhizomes of A. macrocephala were purchased from the Kyungdong oriental herbal market, Seoul, Korea, in October 2015, and identified by one of author (Dr. CY Kim). A voucher specimen 
was deposited in the Herbarium of the College of Pharmacy, Hanyang University (HYUP-AM-001). The dried rhizomes ( $309 \mathrm{~g}$ ) were ground and extracted in $4 \mathrm{~L}$ methanol three times for $3 \mathrm{~h}$ under reflux. The methanol solution was evaporated by rotary evaporator to obtain $46 \mathrm{~g}$ of extract. The extract was suspended in water and successively fractionated by $n$-hexane, ethyl acetate, and $n$-butanol. The fractions obtained were $9.78 \mathrm{~g}$ of $n$-hexane, $6.08 \mathrm{~g}$ of ethyl acetate, and $2.92 \mathrm{~g}$ of $n$-butanol.

\subsection{Selection of the Two-Phase Solvent System}

The two-phase solvent system was selected according to the $K$-values of the target compounds as a series of solvent systems of $n$-hexane-ethyl acetate-methanol-water. Briefly, approximately $2 \mathrm{mg}$ of the sample was added to each test tube, $2 \mathrm{~mL}$ of each phase of a pre-equilibrated two-phase solvent system was added, and the contents were thoroughly mixed. After equilibration, $10 \mu \mathrm{L}$ of the upper and lower phases were analyzed by HPLC at $254 \mathrm{~nm}$. The $K$ value was calculated as the peak area of each compound in the upper stationary phase divided by that of the lower mobile phase.

\subsection{CPC Separation Procedure}

According to the partition coefficient, $n$-hexane-ethyl acetate-methanol-water $(8: 2: 8: 2, v / v)$ was used as the two-phase solvent system for CPC separation. The two-phase system was prepared in a 4000-mL separation funnel by adding $1200 \mathrm{~mL}$ of $n$-hexane, $300 \mathrm{~mL}$ of ethyl acetate, $1200 \mathrm{~mL}$ of methanol, and $300 \mathrm{~mL}$ of water (volume ratio 8:2:8:2). The separation funnel was shaken vigorously and the solvent mixture was thoroughly equilibrated at room temperature. The upper and lower phases were separated and degassed by sonication for $1 \mathrm{~h}$ before use.

First, the rotor was filled with the stationary phase (upper phase), and then the rotor was set at $1100 \mathrm{rpm}$ and the lower, more hydrophilic phase was pumped into the channel at a flow rate of $10 \mathrm{~mL} / \mathrm{min}$ in descending mode. When the stationary phase and mobile phase in the rotor were equilibrated (stationary phase retention: $54 \%$ ), $3 \mathrm{~g}$ of the $n$-hexane fraction dissolved in the $10-\mathrm{mL}$ mixture of stationary phase and mobile phase $(1: 1, v / v)$ was injected. The effluent was monitored at $254 \mathrm{~nm}$ and in scan mode (240-400 nm). Each peak fraction was collected according to the chromatogram to obtain three CPC peak fractions A-C. The fractions were concentrated and analyzed by HPLC.

\subsection{HPLC Analysis and Identification of the CPC Peak Fractions}

The crude extract and each peak fraction obtained by CPC were analyzed by HPLC with an INNO $\mathrm{C} 18$ analytical column $(4.6 \times 250 \mathrm{~mm}, 5 \mu \mathrm{m})$. The mobile phase was composed of acetonitrile containing $0.1 \%$ formic acid (A) and water containing $0.1 \%$ formic acid (B). The gradient elution conditions were as follows: initial 0 min A:B (10:90,v/v), 14 min A:B (25:75), 15 min A:B (43:57), 25 min A:B (43:57), $29 \min A: B(51: 49), 55 \mathrm{~min}$ A:B (51:49), $65 \mathrm{~min}$ A:B (100:0) and $75 \mathrm{~min}$ A:B (100:0). The column temperature was $40{ }^{\circ} \mathrm{C}$, the mobile phase flow rate was $1.0 \mathrm{~mL} / \mathrm{min}$, and the detection wavelength was $254 \mathrm{~nm}$. The injection volume was $10 \mu \mathrm{L}$.

\subsection{Structural Identification}

Identification of the CPC purified compounds was performed by ESI-MS with an Advion compact mass (Advion, Ithaca, NY, USA) and NMR. The ESI-MS spectra conditions were as follows: positive ion mode; mass range, $m / z$ 100-1200; capillary temperature, $200{ }^{\circ} \mathrm{C}$; capillary voltage, $150 \mathrm{~V}$; source voltage offset, 30; source voltage span, 10; source gas temperature, $150{ }^{\circ} \mathrm{C}$; and ESI voltage, $3500 \mathrm{~V}$. ${ }^{1} \mathrm{H}-\mathrm{NMR}(400 \mathrm{MHz})$ and ${ }^{13} \mathrm{C}-\mathrm{NMR}(100 \mathrm{MHz})$ were measured on a Bruker model digital Avance III $400 \mathrm{NMR}$ in $\mathrm{CDCl}_{3}$. The NMR spectra were processed by the MestReNova 9.0 software (Mestrelab Research, Santiago de Compostela, Spain). 


\subsection{Structural Identification of the Purified Compounds}

The structures of 1-3 were identified as atractylenolide I (1), (6E,12E)-tetradeca-6,12diene-8,10-diyne-1,3-diol diacetate (2) and selina-4(14),7(11)-dien-8-one (3) by ${ }^{1} \mathrm{H}$ - and ${ }^{13} \mathrm{C}-\mathrm{NMR}$ data compared with previously published data $[6-8,10]$.

\subsection{Cell Culture and Cell Viability}

Human hepatoma (HepG2) cells were obtained from American Type Culture Collection (ATCC, Manassas, VA, USA) and cultured in a DMEM medium supplemented with 10\% FBS and $1 \%$ penicillin-streptomycin in a humidified atmosphere containing $5 \% \mathrm{CO}_{2}$ in air at $37{ }^{\circ} \mathrm{C}$. For determination of cell viability, $4 \times 10^{4}$ cells/well were seeded in 96-well plates and incubated overnight. Cells were treated for $12 \mathrm{~h}$ with various concentrations of the isolated compounds 1-3. Samples were dissolved in DMSO and the final DMSO concentration did not exceed $0.1 \%$, which did not affect cell cytotoxicity. Cells were then exposed to $0.5 \mathrm{mg} / \mathrm{mL} \mathrm{MTT}$ for an additional $1 \mathrm{~h}$ at $37^{\circ} \mathrm{C}$. The media was removed and $100 \mu \mathrm{L}$ of dimethylsulfoxide was added to dissolve the formazan crystals produced in each well. Absorbance was measured at $570 \mathrm{~nm}$ using an Infinite M200 PRO microplate reader (Tecan, Salzburg, Austria).

\subsection{ARE Luciferase Reporter Assay}

Construction of the HepG2-ARE cells (transfected Pg14.37 [luc2P/ARE/ Hygro] (Promega)) was reported previously [23]. HepG2-ARE cells were cultured in DMEM high glucose media supplemented with 10\% FBS, 1\% penicillinstreptomycin and 1\% hygromycin B. HepG2-ARE cells were plated at a density of $1 \times 10^{5}$ cells / well in 24-well plates and allowed to reach 70-80\% confluence. After $12 \mathrm{~h}$ of serum starvation, cells were incubated with the extract, sub-fractions or isolated compounds 1-3 for $12 \mathrm{~h}$. After treatment, cells were washed twice with ice-cold phosphate-buffered saline, and $120 \mu \mathrm{L}$ of passive lysis buffer (Promega, Madison, WI, USA) was added to each well and reacted at $4{ }^{\circ} \mathrm{C}$ for $30 \mathrm{~min}$. The supernatant was transferred to an e-tube and centrifuged at 13,000 rpm for $15 \mathrm{~min}$ at $4{ }^{\circ} \mathrm{C}$, and $60 \mu \mathrm{L}$ of the supernatant was incubated with $30 \mu \mathrm{L}$ of the luciferase assay substrate (Promega, Madison, WI, USA) in a 96-well plate. Luminescence was measured by an EnSpire multimode plate reader (PerkinElmer, Waltham, MA, USA). Sulforaphane (5 $\mu \mathrm{M})$ (Calbiochem, Darmstadt, Germany) was used as a positive control.

\subsection{Statistical Analysis}

All data are reported as means \pm S.E. The statistical significance of differences between treatments was assessed using the Student's $t$-test. Probability values less than 0.01 were considered significant.

\section{Conclusions}

In this study, bioactivity-guided isolation for ARE-inducing compounds was developed using a combination of CPC and an ARE luciferase reporter assay. One-step CPC enabled the isolation of two sesquiterpenes including atractylenolide I (1) and selina-4(14),7(11)-dien-8-one (3) and one polyacetylene, (6E,12E)-tetradeca-6,12-diene-8,10-diyne-1,3-diol diacetate (2). Among these compounds, compounds 2 and 3 exerted ARE-inducing activity. The overall results of this study demonstrated that CPC is an efficient tool for bioactivity-guided purification from natural products.

Author Contributions: M.I.K. and J.H.K. performed the experiments and wrote the manuscript; A.S.S. participated in carrying out the experiment; Y.-M.K., K.K.C. and C.Y.K. designed and guided the experiment.

Funding: This research was supported by the Basic Science Research Program through the National Research Foundation of Korea (NRF) funded by the Ministry of Science and ICT (NRF-2016R1A2B4014598).

Conflicts of Interest: The authors declare no conflict of interest. 


\section{References}

1. Reuter, S.; Gupta, S.C.; Chaturvedi, M.M.; Aggarwal, B.B. Oxidative stress, inflammation, and cancer: How are they linked? Free Radic. Biol. Med. 2010, 49, 1603-1616. [CrossRef] [PubMed]

2. Xiang, Y.; Haixia, W.; Lijuan, M.; Yanduo, T. Isolation, purification and identification of antioxidants from Lepidium latifolium extracts. Med. Chem. Res. 2018, 27, 37-45. [CrossRef]

3. Surh, Y.J.; Kundu, J.K.; Li, M.H.; Na, H.K.; Cha, Y.N. Role of Nrf2-mediated heme oxygenase-1 upregulation in adaptive survival response to nitrosative stress. Arch. Pharm. Res. 2009, 32, 1163-1176. [CrossRef] [PubMed]

4. Han, H.Y.; Yang, Y.S.; Kim, S.N.; Han, S.C.; Lee, J.H.; Jeong, J.Y.; Roh, H.; Seok, J.H.; Lee, J.S.; Kim, J.A.; et al. Two-week repeated dose toxicity of Atractylodis Rhizoma Alba in F344 rats. Nat. Prod. Sci. 2016, 22, 180-186. [CrossRef]

5. Yun, B.R.; Weon, J.B.; Lee, B.H.; Lee, J.W.; Eom, M.R.; Ma, C.J. Quantitative analysis of atractylenolide I and III in Atractrylodes macrocephala. Kor. J. Pharmacogn. 2013, 44, 53-59.

6. Chang, Y.H.; Kim, C.T.; Jung, M.H.; Lim, Y.H.; Lee, S.H.; Kang, S.J. Inhibition of melanogenesis by selina-4,7-dien-8-one isolated from Atracylodis Rhizome Alba. Biol. Pharm. Bull. 2007, 30, 719-723. [CrossRef] [PubMed]

7. Chen, L.G.; Jan, Y.S.; Tsai, P.W.; Norimoto, H.; Michihara, S.; Murayama, C.; Wang, C.C. Anti-inflammatory and antinociceptive constituents of Atractylodes japonica Koidzumi. J. Agric. Food Chem. 2016, 64, 2254-2262. [CrossRef] [PubMed]

8. Li, X.; Lin, J.; Han, W.; Mai, W.; Wang, L.; Li, Q.; Lin, M.; Bai, M.; Zhang, L.; Chen, D. Antioxidant ability and mechanism of rhizoma Atractylodes macrocephala. Molecules 2012, 17, 13457-13472. [CrossRef] [PubMed]

9. Dong, H.; He, L.; Huang, M.; Dong, Y. Anti-inflammatory components isolated from Atractylodes macrocephala Koidzmi. Nat. Prod. Res. 2008, 22, 1418-1427. [CrossRef] [PubMed]

10. Kano, Y.; Komatsu, K.I.; Saito, K.I.; Bando, H.; Sakurai, T. A new polyacetylene compound from Atractylodes rhizome. Chem. Pharm. Bull. 1989, 37, 193-194. [CrossRef]

11. Yao, C.M.; Yang, X.W. Bioactivity-guided isolation of polyacetylenes with inhibitory activity against NO production in LPS-activated RAW264.7 macrophages from the rhizomes of Atractylodes macrocephala. J. Ethnopharmacol. 2014, 151, 791-799. [CrossRef] [PubMed]

12. Tsai, C.J.; Liang, J.W.; Lin, H.R. Sesquiterpenoids from Atractylodes macrocephala act as farnesoid X receptor and progesterone receptor modulators. Bioorg. Med. Chem. Lett. 2012, 22, 2326-2329. [CrossRef] [PubMed]

13. Walasek, M.; Grzegorczyk, A.; Malm, A.; Skalicka-Woźniak, K. Bioactivity-guided isolation of antimicrobial coumarins from Heracleum mantegazzianum Sommier \& Levier (Apiaceae) fruits by high-performance counter-current chromatography. Food Chem. 2015, 186, 133-138. [PubMed]

14. Foucault, A.P.; Chevolot, L. Counter-current chromatography: Instrumentation, solvent selection and some recent applications to natural product purification. J. Chromatogr. A 1988, 808, 3-22. [CrossRef]

15. Zheng, Z.; Wang, X.; Liu, P.; Li, M.; Dong, H.; Qiao, X. Semi-preparative separation of 10 caffeoylquinic acid derivatives using high speed counter-current chromatography combined with semi-preparative HPLC from the roots of burdock (Arctium lappa L.). Molecules 2018, 23, 429. [CrossRef] [PubMed]

16. Zhang, Y.Q.; Luo, J.G.; Han, C.; Xu, J.F.; Kong, L.Y. Bioassay-guided preparative separation of angiotensin-converting enzyme inhibitory $C$-flavone glycosides from Desmodium styracifolium by recycling complexation high-speed counter-current chromatography. J. Pharm. Biomed. Anal. 2015, 102, $276-281$. [CrossRef] [PubMed]

17. Chen, B.; Peng, Y.; Wang, X.; Li, Z.; Sun, Y. Preparative separation and purification of four glycosides from Gentianae radix by high-speed counter-current chromatography and comparison of their anti-NO production effects. Molecules 2017, 22, 2002. [CrossRef] [PubMed]

18. Budryn, G.; Nebesny, E.; Pałecz, B.; Rachwał-Rosiak, D.; Hodurek, P.; Miśkiewicz, K.; Oracz, J.; Żyżelewicz, D. Inclusion complexes of $\beta$-cyclodextrin with chlorogenic acids (CHAs) from crude and purified aqueous extracts of green Robusta coffee beans (Coffea canephora L.). Food Res. Int. 2014, 61, 202-213. [CrossRef]

19. Zhao, C.; Chaohong, H.E. Preparative isolation and purification of atractylon and atractylenolide III from the Chinese medicinal plant Atractylodes macrocephala by high-speed counter-current chromatography. J. Sep. Sci. 2006, 29, 1630-1636. [CrossRef] [PubMed] 
20. Conway, W.D. Counter-current chromatography: Simple process and confusing terminology. J. Chromatogr. A 2011, 1218, 6015-6023. [CrossRef] [PubMed]

21. Park, H.; Lim, H.; Kim, H.P.; Kwon, Y.S. Downregulation of matrix metalloproteinase-13 by the root extract of Cyathula officinalis Kuan and its constituents in IL-1 $\beta$-treated chondrocytes. Planta Med. 2011, 77, 1528-1530. [CrossRef] [PubMed]

22. Jeong, S.I.; Kim, S.Y.; Kim, S.J.; Hwang, B.S.; Kwon, T.H.; Yu, K.Y.; Han, S.H.; Suzuki, K.; Kim, K.J. Antibacterial activity of phytochemicals isolated from Atractylodes japonica against methicillin-resistant Staphylococcus aureus. Molecules 2010, 15, 7395-7402. [CrossRef] [PubMed]

23. Jeon, J.S.; Park, C.L.; Syed, A.S.; Kim, Y.M.; Cho, I.J.; Kim, C.Y. Preparative separation of sesamin and sesamolin from defatted sesame meal via centrifugal partition chromatography with consecutive sample injection. J. Chromatogr. B 2016, 1011, 108-113. [CrossRef] [PubMed]

Sample Availability: Samples of the compounds 1-3 are from the authors.

(C) 2018 by the authors. Licensee MDPI, Basel, Switzerland. This article is an open access article distributed under the terms and conditions of the Creative Commons Attribution (CC BY) license (http://creativecommons.org/licenses/by/4.0/). 